\title{
Mature Cystic Teratoma of the Suprasternal Fossa in an Adult: Report of Case
}

\author{
Jiangjiang Liu ${ }^{*}$, Xiaojie Ang2\#, Xingpo Guo ${ }^{3 \#, ~ Y u ~ F e n g ~}{ }^{1 \#, ~ H a i t a o ~} \mathrm{Ma}^{1 \dagger}$ \\ ${ }^{1}$ Department of Thoracic Surgery, The First Affiliated Hospital of Soochow University, Suzhou, China \\ ${ }^{2}$ Department of Urology, The First Affiliated Hospital of Soochow University, Suzhou, China \\ ${ }^{3}$ Department of General Surgery, The First Affiliated Hospital of Soochow University, Suzhou, China \\ Email: †mht7403@163.comm
}

How to cite this paper: Liu, J.J., Ang, X.J., Guo, X.P., Feng, Y. and Ma, H.T. (2020) Mature Cystic Teratoma of the Suprasternal Fossa in an Adult: Report of Case. Case Reports in Clinical Medicine, 9, 385-391. https://doi.org/10.4236/crcm.2020.912053

Received: November 23, 2020

Accepted: December 26, 2020

Published: December 29, 2020

Copyright $\odot 2020$ by author(s) and Scientific Research Publishing Inc. This work is licensed under the Creative Commons Attribution International License (CC BY 4.0).

http://creativecommons.org/licenses/by/4.0/ (c) (i) Open Access

\begin{abstract}
Teratomas have been reported to occur in multiple organ regions, and are less common in non-gonadal regions, such as the neck and chest, than in gonadal and midline regions of the body, such as ovaries and testis. Cases have been reported of a large teratoma of the anterior mediastinum extending to the neck, causing symptoms such as dyspnea, which can be quickly detected by Ultrasound, and patients can be quickly treated. In adults, primary teratoma at the suprasternal foss that not accumulate thyroid gland upward and not invade the mediastinum are rare, usually have no obvious clinical symptoms and are found in most patients by chance. In this literature, we report a rare case of mature cystic teratoma in the suprasternal fossa of a 33-year-old male. Preoperative ultrasonography showed a superior sternal fossa tumor with less calcification and more adipose tissue. The final pathologic diagnosis was mature cystic teratoma through open surgery of the suprasternal neck incision. The patient was followed up for 9 months and there was no recurrence. We believe that the suprasternal notch approach is a safe and effective method for the treatment of mature teratoma without protruding into the superior mediastinum.
\end{abstract}

\section{Keywords}

Total Surgical-Resection, Case Report, Suprasternal Fossa, Mature Cystic Teratoma, Adult, Ultrasound

\section{Introduction}

Teratoma is a tumor with the potential of polydermal differentiation, which ori-

${ }^{\star}$ First author.

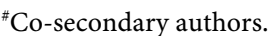

${ }^{\dagger}$ Corresponding author. 
ginates from ectoderm, mesoderm and endoderm in gonads or embryonic leftovers. Most of the tumor tissues are mature and a few are immature [1]. It is usually found in the ovary and testes. In all teratoma reports, the cervical teratomas are rare and often occur in the fetal neck [2]. In adulthood, the occurrence of neck teratoma is extremely rare, accounting for only $10.6 \%$ [3]. The suprasternal fossa, as the entrance of the thorax, is located at the junction of the neck and chest. In the past, some cases of cervical teratomas in adults have been reported. In fact, most of them are cervical extension of anterior mediastinal teratomas due to internal hemorrhage of teratoma or invasion of the space between the cerciothoracic junction or other reasons [4]. Most of them present as midline involvement and are relate to the thyroid gland [5]. Suprasternal fossa is the body surface marker for palpation of the trachea in the neck. Larger tumors pressing on the airway can produce symptoms such as dyspnea and are detected early. However, due to the absence of obvious clinical symptoms and hidden locations, smaller suprasternal teratomas are rarely found. Here, we first report a case of 33-year-old man with suprasternal fossa tumor, which was pathologically confirmed as a primary small and mature cystic teratoma in the suprasternal fossa after surgical resection.

\section{Case Report}

A previously healthy 33-year-old male patient accidentally discovered a mass in the suprasternal fossa without any discomfort. Physical examination: a soft, well-defined mass can be touched in the suprasternal fossa about the size of 40 $\mathrm{mm} \times 20 \mathrm{~mm}$, the degree of activity can be, no movment with swallowing, contacting with no pain. Laboratory examination: CEA: $3.03 \mathrm{ng} / \mathrm{mL}$ and AFP: 2.10 $\mathrm{ng} / \mathrm{mL}$, were normal. Ultrasound examination: A mixed echo can be seen in the subcutaneous soft tissue of the suprasternal fossa, about $43 \mathrm{~mm} \times 22 \mathrm{~mm}$ in size, with regular shape and clear boundaries. Papillary hyperecho and linear hyperecho were found in the cyst wall, and the possibility of cystic teratoma was considered in ultrasonic diagnosis. (Figure 1(a), Figure 1(b)). Neck CT: suprasternal fossa tumor, considering the possibility of suprasternal fossa lipoma (Figure $1(\mathrm{c})$, Figure $1(\mathrm{~d})$ ). The patient refused a proposed magnetic resonance imaging (MRI). His family history was not notable for this disease.

There is no obvious contraindication. Suprasternal fossa tumor resection was performed. During the operation: the mass was located in the suprasternal fossa, the size was about $40 \mathrm{~mm} \times 40 \mathrm{~mm} \times 50 \mathrm{~mm}$, the texture was soft, the boundary was clear, and the cyst was completely peeled off around the cyst wall. The cut surface is cystic with naked eyes, with light yellow oil-like substance inside, hair inside, no obvious heads (Figure 2). Postoperative pathology: (neck) cystic mature teratoma. Immunopathology: CK (epithelial +), CD68 (partial cell +), Ki-67 (scattered +) (Figure 3). The patient recovered well and was discharged three days after operation. There was no recurrence after follow-up for nine months. 


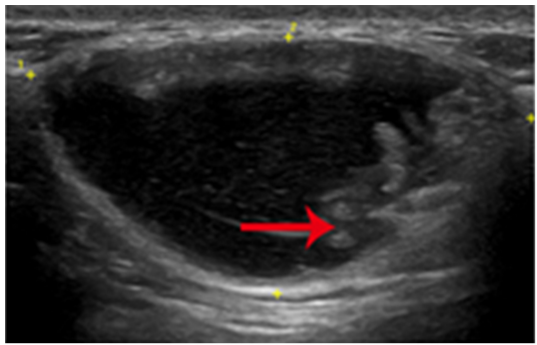

(a)

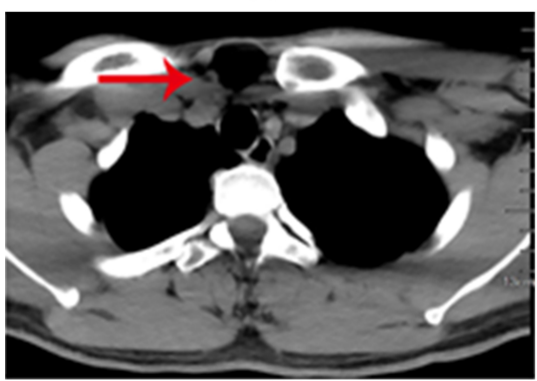

(c)

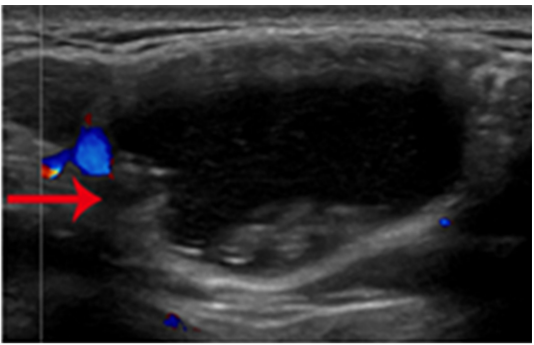

(b)

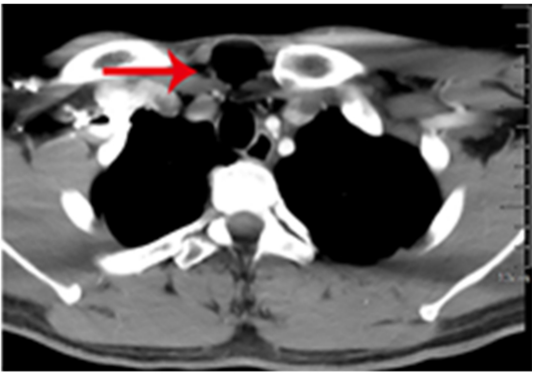

(d)

Figure 1. (a) Ultrasound findings. A mixed echo can be seen in the subcutaneous soft tissue of the suprasternal fossa. The shape is regular, the boundary is clear, and the cystic part has good sound penetration. A papillary slightly higher echo and a line-like strong echo can be seen in the cyst wall. (Be shown by the red arrow); (b) ultrasound CDFI images showed a few blood flow signals during the period. (Be shown by the red arrow); (c) CT plain scan showed that a kind of round low-density focus can be seen in the suprasternal fossa, the trachea was in the center, there was no obvious compression and displacement. (Be shown by the red arrow); (d) enhanced CT, showed that there was no obvious enhancement after contrast-enhanced scan. (Be shown by the red arrow).

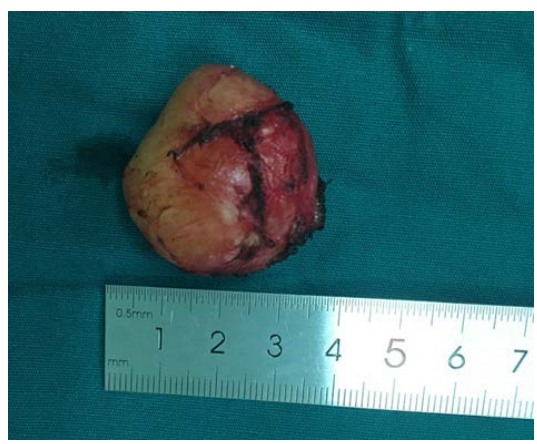

Figure 2. The tumor was completely dissected during the operation.

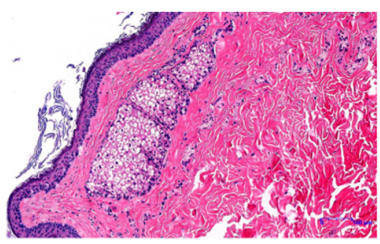

(a)

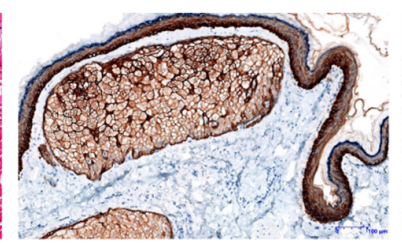

(b)

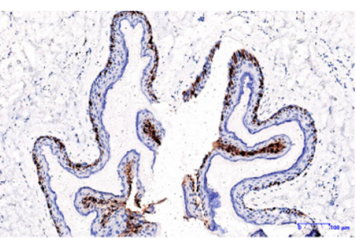

(c)

Figure 3. (a) The cyst wall of the tumor is lined with squamous epithelium, with smooth muscle tissue, sebaceous gland and sweat gland tissue visible in it, and fibrous tissue in the center (HE staining, $\times 200)$; (b) immunohistochemical staining, CD68+ $(\times 200)$; (c) immunohistochemical staining, Ki67+ $(\times 200)$. 


\section{Discussion}

Teratoma is a tumor derived from gonads or embryonic leftovers with polydermal differentiation potential cells, which often occurs in the gonad area and the midline of the body, mostly in the ovary and testis, secondly in the sacral caudal, mediastinum, retroperitoneal and so on [1] [6]. Teratoma can be divided into "mature" and "immature". The distinction is mainly based on the number of immature tissues, especially neurocutaneous tissue [7] [8]. Mature teratoma is more common as a cystic mass, which is composed of well-differentiated adult tumors. It is benign and is called mature cystic teratoma, also known as dermoid cyst [9]. Immature teratoma is composed of immature tissue, which is often solid and malignant, and is more common in teenagers [10]. Teratoma in the neck is extremely rare [6]. Teratomas of the neck account for $1.5 \%$ to $5 \%$ of all sites and often occurs in newborns or infants [7]. Occasionally, cervical teratoma is found in fetal period [10]. Mediastinal teratomas account for 7\% - 9.3\% of mediastinal tumors and 5\% - 7\% of all mediastinal germ cell tumors. Most mediastinal teratomas originate in the anterior mediastinum, with occasional reports of teratomas occurring in the posterior mediastinum [11]. Teratomas of the anterior mediastinum may extend to the neck due to intratumoral hemorrhage, tissue necrosis, infection or tumor invasion, while teratomas of the suprasternal fosse alone have not been reported. The pathogenesis of these tumors in extragonadal sites is likely related to the displacement of primitive germ cells during embryogenesis [11]. Here, we first reported a small mature cystic teratoma, which occurred in the suprasternal fossa, did not move with swallowing, and CT images showed that the tumor did not extend into the mediastinum.

Teratomas extending from the anterior mediastinum to the neck can cause pain or dyspnea due to internal bleeding or infection, while microcystic teratomas with suprasternal fosse are usually asymptomatic [12], so there are some difficulties in detection, diagnosis and treatment of the disease at an early stage. Ultrasonography and CT showed clear round or quasi-round thick-walled hyperechoic masses and uneven internal density, especially for thick-walled cysts with oil, hair, bone tissue, teeth and calcification, which were often characteristic manifestations of teratoma [13]. The CT density of cystic mature teratomas is often close to water, because less calcified tissue and more adipose tissue in the tumor [4]. Fat-fluid levels are diagnostic of teratoma but are seen less frequently [14], which could be used as one of the diagnostic criteria of teratoma [3] [6] [10] [15]. Therefore, when encountering teratomas with such imaging manifestations, teratoma should be differentiated from lipomas. Computed tomography can also determine the encroachment of adjacent structures and aid in surgical planning [16]. Magnetic resonance imaging showed high T1 and T2-weighted signals in teratomas, low T1 and T2-weighted signals in fat suppression, with hair, bone and other tissue signals are also of low intensity [17]. Different types of teratomas have different tissue types and internal components, so they are heterogeneous on T1 and T2 weighted images [18]. Combined with this case, ultrasound 
examination showed that a cystic mixed echo mass could be seen in the subcutaneous soft tissue, with regular shape, clear boundary, papillary high echo and linear strong echo in the capsule wall. CT revealed a round low-density lesion in the suprasternal fossa with no obvious calcification. AFP is normal. In contrast, immature teratoma showed solid and irregular parenchyma calcification on ultrasound, extensive parenchyma calcification could be seen on CT, and AFP value was significantly increased. Teratoma with more cystic components often needs to be differentiated from thyroid cystic space occupying, cystic hydroma, branchial cleft cyst, thyroglossal duct cyst, cervical echinococcosis, etc. Teratoma with more solid components should be differentiated from cervical lipoma, lymphoma, thyroid adenoma and so on [19].

Neville P. Shine et al. found that the malignant probability of congenital neck teratoma is less than 5\% [7]. The principle of treatment is to remove the focus completely. Once the cervical mature cystic teratoma is found, the mass and adherent tissue should be removed. If there is a fistula, the fistula should be eliminated to prevent recurrence and malignant transformation. Early and thorough resection has a good prognosis [3] [10]. The scope of surgical resection must be based on rapid pathological examination during the operation. If it is benign, the tumor and its appendages can be removed. If it is malignant or the lesion grows rapidly within a short period of time, and malignant changes are suspected, the scope of surgical resection should be expanded. Unlike teratomas with mediastinal extension to the neck, primary mature cystic teratomas of the suprasternal fossae require surgery only through the suprasternal transection of the neck, rather than through the thorax. As of this report, this case has been followed up for nine months and there are no signs of recurrence. Some scholars believe that plasma AFP can be used as a follow-up index after operation. The half-life of AFP in recurrent tumor is longer than that in non-recurrent teratoma, and the half-life of AFP in immature teratoma is longer than that in mature teratoma [20] [21]. Considering the wishes of the patient, the plasma AFP was not detected during the postoperative follow-up. And the plasma AFP was normal before operation, which may be related to the nature of mature cystic teratoma.

\section{Conclusion}

Suprasternal fossae is an ectopic site of thoracic teratomas. This case highlights the rare primary site of cervical and thoracic teratomas in adults. The preliminary diagnosis can be obtained by preoperative imaging examination and serum tumor marker examination. It can be concluded from this report that ultrasound is the first preoperative examination choice for this disease. At present, complete surgical resection via suprasternal notch is the safest and effective method for the treatment of mature teratoma without protruding into the superior mediastinum, and the prognosis is generally good.

\section{Data Availability}

The data used to support this study are restricted to protect patient privacy. 


\section{Patient Consent for Publication}

Written informed consent for publication of the present report was obtained from the patient.

\section{Availability of Data and Materials}

The datasets used and/or analyzed during the present study are available from the corresponding author on reasonable request.

\section{Authors' Contributions}

Haitao Ma conceived and designed this case report. Jiangjiang Liu is the first author, wrote the initial draft of the report. All authors have read and approved the final version of the manuscript.

\section{Ethics Approval and Consent to Participate}

Written informed consent for surgery was obtained from the patient.

\section{Conflicts of Interest}

The authors declare no conflicts of interest regarding the publication of this paper.

\section{References}

[1] Wolter, N.E., Siegele, B. and Cunningham, M.J. (2017) Cystic Cervical Teratoma: A Diagnostic and Management Challenge. International Journal of Pediatric Otorhinolaryngology, 95, 97-100. https://doi.org/10.1016/j.ijporl.2017.02.016

[2] Alexander, V.R., George Manjaly, J., Pepper, C.M., Ifeacho, S.N., Hewitt, R.J. and Hartley, B.E.J. (2015) Head and Neck Teratomas in Children-A Series of 23 Cases at Great Ormond Street Hospital. International Journal of Pediatric Otorhinolaryngology, 79, 2008-2014. https://doi.org/10.1016/j.ijporl.2015.07.042

[3] Deng, H.Y., Wang, Z.Y., Yang, Q.T. and Ye, J. (2020) Mature Cervical Teratoma Extending into the Anterior Mediastinum of an Adult. Ear, Nose \& Throat Journal. https://doi.org/10.1177/0145561320925563

[4] Agarwal, G. and Kar, D.K. (2008) Teratoma of the Anterior Mediastinum Presenting as a Cystic Neck Mass: A Case Report. Journal of Medical Case Reports, 2, Article No. 23. https://doi.org/10.1186/1752-1947-2-23

[5] Win, T.T., Fakarul Razy, N.H.M., Ab Hamid, S.S., Balas Ubramanian, A. and Ramalinggam, G. (2014) Congenital Mature Cystic Teratoma of the Lateral Neck Presenting as Cystic Hygroma: A Rare Case Report with Literature Review. Turkish Journal of Pathology, 30, 220-224. https://doi.org/10.5146/tjpath.2013.01220

[6] Wakhlu, A. and Wakhlu, A.K. (2000) Head and Neck Teratomas in Children. Pediatric Surgery International, 16, 333-337. https://doi.org/10.1007/s003830000391

[7] Shine, N.P., Sader, C., Gollow, I. and Lannigan, F.J. (2006) Congenital Cervical Teratomas: Diagnostic, Management and Postoperative Variability. Auris Nasus Larynx, 33, 107-111. https://doi.org/10.1016/j.anl.2005.07.003

[8] Kim, H.J and Koh, Y.S. (2019) Mature Cystic Teratoma of the Pancreas: A Rare Cystic Neoplasm. Open Medicine, 14, 872-874. https://doi.org/10.1515/med-2019-0102 
[9] Zhou, Y.C, Pan, C.C., Guo, T., Wang, Y.F., Zhao, Y. and Sun, J.W. (2020) Endonasal Transpterygoid Surgery for Adult Mature Teratoma in the Sphenoid Bone. Journal of Craniofacial Surgery, Publish Ahead of Print. https://doi.org/10.1097/SCS.0000000000006884

[10] Herman, T.E. and Siegel, M.J. (2008) Cervical Teratoma. Journal of Perinatology, 28, 649-651. https://doi.org/10.1038/jp.2008.84

[11] Ibi, T., Hirai, K., Takeuchi, S., Bessho, R., Kawamoto, M., Shuji, H., et al. (2013) Mature Teratoma of the Posterior Mediastinum: Report of a Case. General Thoracic and Cardiovascular Surgery, 61, 655-658. https://doi.org/10.1007/s11748-012-0168-2

[12] Alimehmeti, M., Alimehmeti, R., Ikonomi, M., Saraci, M. and Petrela, M. (2013) Cystic Benign Teratoma of the Neck in Adult. World Journal of Clinical Cases, 1 , 202-204. http://dx.doi.org/10.12998/wjcc.v1.i6.202

[13] Kadlub, N., Touma, J., Leboulanger, N., Garel, C., Soupre, V., Coulomb L'Herminé, A., et al. (2014) Head and Neck Teratoma: From Diagnosis to Treatment. Journal of Cranio-Maxillofacial Surgery, 42, 1598-1603. https://doi.org/10.1016/j.jcms.2014.04.028

[14] Rosado-de-Christenson, M.L., Templeton, P.A. and Moran, C.A. (1992) From the Archives of the AFIP. Mediastinal Germ Cell Tumors: Radiologic and Pathologic Correlation. RadioGraphics, 12, 1013-1030. https://doi.org/10.1148/radiographics.12.5.1326777

[15] Peiró, J.L., Sbragia, L., Scorletti, F., Lim, F.Y. and Shaaban, A. (2016) Management of Fetal Teratomas. Pediatric Surgery International, 32, 635-647. https://doi.org/10.1007/s00383-016-3892-3

[16] Nichols, C.R. (1991) Mediastinal Germ Cell Tumors. Clinical Features and Biologic Correlates. CHEST, 99, 472-479. https://doi.org/10.1378/chest.99.2.472

[17] Barth, A., Nassenstein, I., Tröbs, R.B., Tannapfel, A., Dercks, H., Rostásy, K., et al. (2019) N-Methyl-D-Aspartate Receptor Encephalitis with Psychiatric Symptoms and an Ovarian Teratoma Detected by MRI in a 17-Year-Old Girl. Neuropediatrics, 50, 253-256. https://doi.org/10.1055/s-0039-1692417

[18] Wang, H., Huang, Y., Nie, P., Dong, C., Hou, F., Hao, D. and Xu, W. (2016) MRI Findings in Intraspinal Mature Teratoma. Clinical Radiology, 71, 717.E1-717.E8. https://doi.org/10.1016/j.crad.2016.04.010

[19] Bojino, A., Emanuele, Z., Alessandro, G. and Giovanni, G. (2020) Surgical Treatment of Massive Teratoma of the Orbit. Ophthalmic Plastic and Reconstructive Surgery, 36, e68-e70. https://doi.org/10.1097/IOP.0000000000001581

[20] Elmasalme, F., Giacomantonio, M., Clarke, K.D., Othman, E. and Matbouli, S. (2000) Congenital Cervical Teratoma in Neonates. Case Report and Review. European Journal of Pediatric Surgery, 10, 252-257. https://doi.org/10.1055/s-2008-1072369

[21] Nam, S.H., Cho, M.J., Kim, D.Y. and Kim, S.C. (2018) Half-Life of Alpha-Fetoprotein in Neonatal Sacrococcygeal Teratoma. Journal of Pediatric Surgery, 53, 2470-2474. https://doi.org/10.1016/j.jpedsurg.2018.08.012 